\title{
Training strategies of reverse thinking in poetry writing teaching
}

\author{
Qizhen Li \\ Hubei University of Science and Technology. Xianning Hubei ,437005, China
}

Keywords: Poetry writing, Reverse Thinking, Training strategies.

\begin{abstract}
As an important discipline in junior middle school, Chinese plays a basic role in the whole education system. While, writing is an important module of the whole Chinese language teaching. It is the comprehensive performance of students' literacy and language ability as well as an important way to show the quality of students' thinking. As the focus of the whole writing module, there is a deep personal emotion in poetry writing and it is a unique manifestation of personalized creation. Therefore, poetry writing must follow the principle of lofty conception and unique insight. Thus it is very important to cultivate students' creative thinking in poetry writing training. Only by looking at the world in a particular way, can we have our own way of thinking in writing and write valuable and creative articles. Among many ways of training students' thinking, reverse thinking is to think from the opposite direction and arouse students' interest. And it is necessary to establish the habit of reverse thinking, to achieve surprise effect. This paper is based on the various types of literature theoretical analysis of reverse thinking method, and discusses the current existing problems in the teaching of poetry writing then puts forward the effective strategies of reverse thinking, hoping it is helpful for poetry writing.

The reverse thinking is also called "thinking of seeking difference". As an important form of expression in many creative thinking, it has the unique characteristics of revolutionary, metabolic, distinctive and innovative[1].The practice results show that the reverse thinking is helpful to cultivate students' thinking ability, and it has a profound influence on improving individual's comprehensive creative ability and solving practical problems ability, especially in poetry writing. Reverse thinking itself is critical and novel. From the research traits, reverse thinking and positive thinking are relative. The positive is to think about the usual, accepted and habitual perspectives and ideas when facing problems. While the reverse is just the opposite. It is rebellion of the traditional thinking. It is required to challenge conventional ideas , think in a different way and not be stick to the rules.It is helpful for students to overcome the mindset and break the rigid thinking mode created by experience and habit. Novelty is the embodiment of the direction of thinking. It guides students not to solve problems in accordance with the rules and regulations and abandon the habit of thinking summed by the past experience and not to be influenced by the traditional methods. Because people tend to see their familiar side and think in an experienced way, giving up the other side of obstacles. In this way, they can not get unexpected conclusions and make refresh changes. Reverse thinking is thinking from the other side. Poetry creation requires novelty, criticism and creativity.
\end{abstract}

\section{An introduction of the types of reverse thinking}

Backward thinking method. Shu Ting's "The Goddess Peak" is a typical representative poetry of this type of thinking method, it was written by reverse thinking method. When you use this method to understand something, you need to start from the opposite direction and think about the other side. Usually, it focuses on the function, structure and causality of things[2]. "The Goddess Peak" represents the heroic and stirring of Chinese women, who is in the detention of traditional ethical but still love until death. "The Goddess Peak" written by Shu Ting did not reflect the praise of the loyalty and tenacity of women in male dominated society. Instead, it uses backward thinking 
method to show the yearning and call for the true nature of life behind loyal love. Especially the last line of poetry echoing the title "It's better to cry for a night on your lover's shoulder", expressed the helpless and pain in a touching love. Abandoning the goddess beautiful coat, revealing the inner voice, exiling women pursuit and declaration of the essence of life freedom and liberation. The poet did not write in a traditional way to express that the female are the victim of the traditional society. He profoundly reveals that the greatest weakness of secular culture is lack of human concern. Reached that it is not a poignant love, but a heavy yoke placed on women. The use of this thinking opens up a new way of thinking for the world. "The Goddess Peak" is lovely and speculative in her words.

Transformation thinking. This kind of thinking is a change of the angle in studying problems. Because of the different angles of view, different thinking experiences will be produced. If the conventional means of solving a problem are hindered, instead of other means or thinking angle, then the problem can be solved smoothly. Such as the history of popular education story - Sima Guang hit the tank. In essence, it is to solve the difficult problems with the transformation thinking method. The traditional thinking of saving the drowning child is to get the child out of the water tank, but because of the height shortage, Sima Guang change the means of saving. He broke the water tank and saved the child, thus solved the problem smoothly. There are many ancient Chinese poetry used the transformation thinking. Another example is Tang Dynasty poet Du Mu's "Chibi".The intention is that the poet dug out a break spear buried for a long time from the sand. However, he did not directly write the story that Zhou Yu beat Cao Cao with the help of the east wind in the battle named Chibi in accordance with traditional thinking. While, he expressed the sense of nostalgia and sorrowful from the ending of the Qiao sisters in Dongwu[3].Transformation thinking helps to interpret poetry from different perspectives and diverge thinking for writing to create more creative works.

Shortcomings thinking method. It's a thinking method requires finding the shortcomings of things in order to turn them into advantages then taking advantage of these shortcomings. This kind of thinking is not an improvisation to seize the shortcomings of things. On the contrary, it turns invisible to be visible and finds the direction of thinking in the predicament. For example, metal corrosion destroys the original properties of metals, but people use the principle of metal corrosion to produce the required metal powder, which is used in electroplating and other uses. This is the essence of shortcomings thinking "A Song of White Snow on Secretary Wu's Return to Capital” written by poet Censen in Tang Dynasty is such a poetry created with this kind of thinking. The poet is intended to describe the cold in northwest China in August, exaggerating the bitter life of the soldiers beyond the Great Wall. But the use of poetry popular words "As though a gust of spring wind swept past overnight, Bringing thousands upon thousands of pear trees into bloom "implicates that there is a beautiful scenery in the extreme cold weather. It makes people feel happy inside and achieve the effect of "bringing the dying back to life". And the poem "Farewell to Mr Du for His Taking Office in Shuzhou "written by Wang Bo in Tang Dynasty is the similar. The original is the sorrow of parting, then shocked by the momentum of "Long distance separates no bosom friends". It encourages friends to pursuit their dreams without caring about the distance, creating a far-reaching and open artistic conception and becoming popular through the ages[4].

I illustrated the poems for the three types of reverse thinking. And concluded that the writing of poems does not only stay in the theme of poetry and the expression of art.It's the internal thinking mode we discovered through the analysis of the overall implication in a poetry. Excellent poetry often has its unique way of thinking. So the teaching of poetry writing should focus on reverse thinking method for training, guide the students using the reverse thinking effectively and learn to master the connotation of thinking then use it in the specific creation.

\section{Problems in the teaching of poetry writing}

Pay more attention to quantity than quality. In the teaching of poetry writing, teachers pay too much attention to the students' requirements of quantity, while ignore the strict quality control of 
students' writing. They did not give effective guidance to students' articles, which led many students to be unclear about their composition problems. For example, many teachers like to analyze the artistic conception created by poetry in the teaching of poetry writing. They create the situation and make students experience the main idea, and then start writing. They did not focus on the way of thinking and the poet's writing ideas to guide students, which makes the number of students writing more, but did not grasp the poetry of writing thinking and writing methods.

Emphasizing textbooks and neglecting practice. After the new curriculum reform, the teachers are still bound by the traditional teaching thought in the daily poetry writing teaching process. They only in accordance with the curriculum standards or teaching plan to teach students knowledge, implementing teaching tediously. It takes too much time to explain the textbook. Only in the poetry class can they be trained, they not get effective guidance in the poetry writing after class. Students are not enthusiastic about writing. Moreover, poetry does not exist in daily life, and requires specific cognition to train students. Poetry writing is to reflect the core of the students' language literacy. Therefore, teachers should try their best to create the artistic conception of poetry writing in Teaching. Textbooks should not be used as the only teaching materials for poetry writing.

Pay more attention to training than cognition. Due to the difficulty of poetry writing, teachers should take into account the way of thinking and thinking habits of students in training, not only implement the teaching plan in accordance with their own teaching schedule. Compared with material writing, poetry is far away from students' life. It is difficult for them to form their own opinions on their life experience and practical cognition. This brings difficulties to writing. The more successful writing teaching is that the teacher inspires the students to understand the things of life according to the students' age cognition level and guide students to use imagination to express their views.At present, most of the teaching mode still adopts the unified teaching method. Teachers lack the training of students' imagination ability, and students can't start with poetry writing and can't think effectively.

\section{Effective strategies of reverse thinking in poetry writing teaching}

Changing the angle of view and writing the other side of things. Dialectics in philosophy tells us that everything in the world is unity of opposites, and both sides are in contradictory unity. We tend to recognize one aspect of things and are accustomed to ignorance of the other. In the teaching of poetry writing, teachers should innovate traditional teaching concepts and break through the traditional thinking set and frame. It is necessary to express the theme of the poetic meaning and not to ignore the poet writing thinking teaching. Although the conventional thinking is difficult to break, but with the three types of reverse thinking methods introduced in the classroom by teachers, students will continue to be accustomed to this way of thinking and gradually learn to use.

Looking at the problems with the development of connections, and writing the changes of things. The reverse thinking method in the philosophy itself is contradictory viewpoint of content, also known as divergent thinking, seeking innovation. Therefore, in the teaching of poetry writing, with the continuous innovation of the writing point of view, we should take into account all aspects of the interpretation of poetry. Because of the reverse thinking itself belongs to a kind of creative thinking, it has unique thinking expansion. It can develop and perfect its theoretical system, and pursue the profound characteristics of thinking. It helps the creator see the essence through the phenomenon. Therefore, the development of contact point of view helps to discover the details of things, stimulate the sensitivity of thinking, and constantly capture new things, transferring the thinking of this kind of objects to another category of objects.

Seeing the essence through the phenomenon and be good at identify false then writing its' essence. The illusion is the essence of the appearance in special circumstances, reflecting the essence in a tortuous form. "Make a feint to the East but attack in the west" in the military is false, its purpose is to destroy the enemy and protect themselves. This is the "Nothing is too deceitful in a war" strategy[5].Transferred to the teaching of poetry writing is the application of the shortcomings thinking method. The essence of things is not manifested in the phenomenon. From a traditional 
point of view, metal rust is the appearance of metal erosion, but the transfer to the essence is that it can be applied to electroplating. To get the surprise effect is to dare to reverse thinking. The real great poetry is the poet's unique way of thinking and there is ideological connotation of the times in their creations, not just meditates on the past and describes things in nature.

\section{Conclusion}

In summary, Reverse thinking mode is not only a simple skill training, but a kind of creative thinking. It can excavate students' creative potential and break the conventional thinking inertia. Zhu Ziqing once said, "the value of classical training is not practical, but in culture". Therefore, teachers should break through the conventional teaching method and use the reverse thinking method to inspire poetry "exception" observation in the teaching of poetry writing, laying a solid foundation for writing and making personalized creation really.

\section{Reference}

[1] Wu Xinchun. Research on the Application of Convergent Thinking and Reverse Thinking in Composition Teaching of High School[D].Sichuan Normal University,2016.

[2] Li Xiang. Reverse thinking, reverse conception[J].Today high school student,2015,(Z5):26-29.

[3] Liu Lifeng. Paying Attention to the Cultivation of Reverse Thinking Mode in the Teaching of Poetry Writing[J].Journal of Changchun Teachers College,2012,31(07):136-138.

[4] Kan Yingbi. A Practical Study on Cultivating Middle School Students' Use of Textbooks for Reverse Thinking Writing[J].Science education,2010,16(04):32-33.

[5] Chen Shiming. Hear me sing the refurbished “A willow branch”-On Reverse Thinking and Poetic Creation (II)[J].The Writing,2008,(13):6-8. 